\title{
Proposta de Avaliação Prática de Frameworks para a Distribuição de Redes de Aprendizado Profundo
}

\author{
Ana Luisa Veroneze Solórzano, Lucas Mello Schnorr \\ Instituto de Informática, Universidade Federal do Rio Grande do Sul (UFRGS) \\ Caixa Postal 15.064 - 91.501-970 - Porto Alegre - RS - Brasil \\ alvsolorzanodinf.ufrgs.br, schnorreinf.ufrgs.br
}

\begin{abstract}
Resumo. Frameworks para distribuir o treinamento de aprendizado profundo usam estruturas aplicáveis a sistemas de computação de alto desempenho, e propõe fácil utilização pelo usuário. Um dos principais desafios dos frameworks é escalar o treinamento para múltiplos processadores, como CPUs e GPUs, sem perder desempenho e precisão com a sobrecarga de comunicações. Este trabalho propõe uma avaliação prática de frameworks recentes tais como Horovod, LBANN e Tarantella para distribuição de Redes de Aprendizado Profundo.
\end{abstract}

\section{Introdução}

Redes Neurais Artificiais usam modelos matemáticos para lidar com problemas reais com processamento similar a capacidade humana de realizar tarefas [Laine 2003]. Assim como o cérebro humano, Redes Neurais Artificiais Profundas também precisam aprender a partir de dados desconhecidos. Essa rede é composta por camadas compostas por neurônios artificiais que se comunicam entre si para obter a melhor previsão ao final.

O treinamento de uma rede neural pode levar dias, dependendo do modelo e dos dados utilizados. Ambientes de computação de alto desempenho são essenciais para processar aplicações como essas, que lidam com grandes quantidades de dados e executam computações exaustivas. Frameworks para distribuir o treinamento de redes de aprendizado profundo facilitam a utilização de múltiplos dispositivos como CPUs, GPUs e FPGAs [Ben-Nun and Hoefler 2019]. Porém, ao implementar abordagens paralelas, essas ferramentas devem preservar aspectos da rede, como garantir que o modelo esteja acessível em todos processos, que os processos tenham os mesmos parâmetros de inicialização, e que todos processos tenham os resultados de outros durante cada época de treinamento. Com isso, o maior desafio destas ferramentas está em escalar o treinamento para mais recursos computacionais e minimizar os custos de computações extra.

Horovod [Sergeev and Balso 2018], LBANN [Van Essen et al. 2015] e Tarantella [CC-HPC 2020] são alguns dos frameworks mais recentes para sistemas com CPUs e GPUs. Eles se propõem a facilitar a distribuição do treinamento utilizando poucas linhas adicionais de código. Acredita-se que com uma avaliação prática dessas ferramentas seja possível comparar o desempenho entre elas, identificando os algoritmos utilizados para distribuição e gargalos nas estratégias implementadas. Este estudo também pode auxiliar na decisão de qual framework utilizar, além de que otimizações no treinamento de Redes Neurais Artificiais de Aprendizado Profundo podem acelerar diversas pesquisas que lidam com grandes modelos e grandes conjuntos de dados. 


\section{Metodologia}

Os experimentos serão conduzidos na Grid' 5000 [Cappello et al. 2005] e no Parque Computacional de Alto Desempenho $\rrbracket^{1}$ utilizando GPUs com Tensor e CUDA cores. Será utilizada uma rede neural convolucional com o dataset FashionMNIST e CIFAR-10 para classificação de imagens. A primeira etapa consiste na definição de um projeto experimental que considere fatores/níveis relevantes para configuração dos treinamentos [Jain 1991]. A segunda etapa consiste em selecionar os melhores casos da primeira, aprofundando a análise com instrumentação do código dos frameworks com Score-P [Knüpfer et al. 2012]. Os rastros coletados permitirão identificar as fases das execuções levando a propostas de melhorias na implementação dos frameworks. A análise com visualização de dados será feita na linguagem $\mathrm{R}$ com pacotes da coleção tidyverse $\mathrm{f}^{2}$. Os frameworks com suporte CPU/GPU serão o Horovod, o LBANN e o Tarantella.

\section{Considerações Finais}

Ao final deste trabalho, espera-se entender, por exemplo, como os frameworks distribuem computações entre diferentes processos, quais estratégias são implementadas para paralelização e quais algoritmos são utilizados para sincronização entre processos. Espera-se propor melhorias na implementação de pelo menos um framework, tal como otimizações na distribuição de carga entre recursos, avaliando e comparando o desempenho entre as ferramentas. Serão criadas visualizações para análise dos rastros, melhor percepção sobre as fases de processamento e identificação de gargalos.

\section{Referências}

Ben-Nun, T. and Hoefler, T. (2019). Demystifying parallel and distributed deep learning: An in-depth concurrency analysis. ACM Comput. Surv., 52(4).

Cappello, F. et al. (2005). Grid'5000: a large scale and highly reconfigurable grid experimental testbed. In The 6th IEEE/ACM International Workshop on Grid Computing, 2005., page 8, Washington, US. IEEE.

CC-HPC, C. (2020). Tarantella: Distributed deep learning framework. http: / / www . tarantella.org. Accessed: 2020-11-14.

Jain, R. (1991). The art of computer systems performance analysis: techniques for experimental design, measurement, simulation, and modeling. Wiley New York.

Knüpfer, A. et al. (2012). Score-P: A Joint Performance Measurement Run-Time Infrastructure for Periscope,Scalasca, TAU, and Vampir. In Brunst, H., Müller, M. S., Nagel, W. E., and Resch, M. M., editors, Tools for High Performance Computing 2011, pages 79-91, Berlin, Heidelberg. Springer Berlin Heidelberg.

Laine, A. (2003). Neural Networks, page 1233-1239. John Wiley and Sons Ltd., GBR.

Sergeev, A. and Balso, M. D. (2018). Horovod: fast and easy distributed deep learning in TensorFlow. arXiv preprint arXiv:1802.05799.

Van Essen, B., Kim, H., Pearce, R., Boakye, K., and Chen, B. (2015). LBANN: Livermore big artificial neural network hpc toolkit. In Proceedings of the Workshop on Machine Learning in HPC Environments, MLHPC '15, New York, NY, USA. ACM.

\footnotetext{
${ }^{1}$ http://gppd-hpc.inf.ufrgs.br/

${ }^{2}$ https://www.tidyverse.org/
} 